\title{
Revising a Model of Crime and Punishment
}

\author{
Ariel Arbiser ${ }^{1}$ \\ ${ }^{1}$ Dpto. de Computación, Facultad de Ciencias Exactas y Naturales - Universidad de \\ Buenos Aires - Buenos Aires - Argentina \\ arbiser@dc.uba.ar
}

\begin{abstract}
We study a model of society consisting of agents and a government interacting according to decisions regarding cost-effective punishment of crime. We evaluate different strategies for the government in order to reduce the criminal activity, considering degrees of honesty and a possible cost for monitoring and apprehension. We extend a previous model by introducing a contagion effect for the degrees of honesty, and study properties which may lead to a crime-free society, including a game-theoretic formulation.
\end{abstract}

\section{Introduction}

In [Becker 1968], a rational study of the illegal act and punishing crimes in a society from an economical standpoint is introduced. Since then, it has been an interesting subject among different academic fields, and it has given important contributions that help to understand how citizens' and governments' rules interact as a consequence of applied political decisions. The models proposed by several authors describe numerous scenarios of real-life problems. Van Eeghen and Fernandez [Van Eeghen 2016] combine models where a game-theory approach is used for deriving the optimal strategy for the tax administration. [Friedland 1978] shows a simulated environment where agents make hypothetical decisions on tax evasion, achieving useful evidence for further theoretical analysis. In [Aprea 2018] we explore the model of crime and punishment dynamics of [Iglesias 2012], we perform computer simulations with different apprehension policies and criteria, and identify utility functions and equilibria in an associated game. In this note, we extend that study mainly with the introduction of leadership and contagion.

\section{Agents and dynamics with possible leadership}

We introduce some generalizations of [Iglesias 2012]. Our population has a fixed number $N$ of independent agents, each one with his monthly income (or wage) and a predisposition to commit a crime given by his degree of honesty. Their behaviour will probably depend on their salaries, the booty opportunity presented, and the risk of capture. If apprehended, the agent is punished with prison time and his wealth is reduced. (All references to monthly events will just denote fixed time instants.) In the cited model, each agent receives a monthly random wage $W_{i} \in\left[W_{\min }, W_{\max }\right]$ as his only source of income together with the booties from successful crimes. Each agent is characterized by a degree of honesty $H_{i}$, which quantifies his inclination to abide by the law. The procedure is summarized as follows: - An offender $i$ is selected among free agents (i.e., not in jail) at random. This agent earns wage $W_{i}$ and has degree of honesty $H_{i}$. The booty $\mathrm{S}$ is estimated as $\gamma K\langle W\rangle(\langle X>$ denotes average of some $X$ values $)$, where $\gamma \in[0,1]$ is a random number and $K$ is a constant. - The probability of apprehension can be given by (1), although we generalize this function. $\bullet$ The expected 
utility $U$ for the agent is given by (2) or (2'). - Agent $i$ commits the crime if $U>0$ (the model ignores certain aspects like moral factors, partially represented by the $H_{i}$ 's). $\bullet$ If $i$ is not punished, he adds $S$ to his wealth. Otherwise, he spends $\tau=1+(S /<W>)$ months in jail (without salary), and his wealth is reduced by $\left(1+f_{D}\right) S$. Typical values are $f_{D}=1 / 4$ and $\pi$ a logistic function (1) with parameters $p_{0}, p_{1}$ :

$$
\begin{gathered}
\pi(S)=p_{1}+\frac{\left(p_{0}+p_{1}\right)}{1+e^{\frac{S-S S}{S S}}} \\
U=(1-\pi(S)) \cdot\left(S+\tau \cdot W_{i}\right)-\pi(S) \cdot\left(1+f_{D}\right) \cdot S-H_{t}-\tau \cdot W_{t}
\end{gathered}
$$

We also generalize the honesty dynamics of the existing model. $H_{i}{ }^{t}$ will denote the degree of an agent $i$ at the time $t$, with a monthly update for all agents as in (3), where $N_{k}{ }^{t}$ is the number of crimes committed at $t, N_{p}{ }^{t}$ is the number of crimes punished at $t$, and $\delta H_{i}>0$ is a constant for the agent (maximum possible change for $H_{i}$, typically 10):

$$
H_{i}^{t+1}=\alpha_{i} H_{i}^{t}+\left(1-\alpha_{i}\right) H_{l(i, t)}^{t}+\delta H_{i}\left(c N_{p}^{t}-N_{k}^{t}\right) / N_{k}^{t}
$$

Integer constant $c>1$ denotes the inverse proportion of punished crimes affecting decisions. We introduce a form of contagion effect by a convex combination of $i$ 's degree of honesty with the one of agent $l(i, t)$, his leader at time $t$, where $0 \leq \alpha_{i} \leq 1$. [Iglesias 2012] adopts the utility of crime given by (2) but we take the more general

$$
U=(1-\pi(S)) \cdot\left(S+\tau \cdot W_{i}\right)-\pi(S) \cdot(S+f(S))-H_{i}-\tau \cdot W_{i}
$$

where $f(S)$ is the fine that an offender pays when stealing the booty $S$, and we let $\pi$ be any probability distribution. The agent chooses to commit a crime iff $U>0$. Iglesias et al. take $c=2, \pi$ a logistic function, all $\delta H_{i}$ equal and $f(S)=f_{D} \cdot S$ where $f_{D}>0$ is fixed.

\subsection{Agent behavior}

We still note that there is a critical probability value at which any agent will abstain from committing a crime, ignoring any other variable in equation (2').

Lemma 1. Given any distribution $\pi: \mathfrak{R}_{\geq 0} \rightarrow[0,1], \mathrm{f}: \mathfrak{R}_{\geq 0} \rightarrow \mathfrak{R}_{\geq 0}, \tau, W_{i}, H_{i} \geq 0$, if for all $S>0 \pi(S) \geq S /(2 S+f(S))$ then the agent does not commit a crime. In particular, if for all $S>0 \pi(S) \geq 1 / 2$, then the agent does not commit a crime.

The global bound $1 / 2$ can be changed by means of conditioning $f$ (see [Aprea 2018]).

Let us comment about the minimum probability of capture to avoid crime. Running simulations for a $\pi$ with a lower bound of $1 / 2$ or higher is not useful because no crime will be committed. Furthermore, by fixing $f_{D}=1 / 4$ for instance, the critical value at which an agent won't commit a crime based solely on $\pi$ is obtained from Lemma 1 . If no crime is committed from time $t$, we will assume that the criminal behaviour of all agents disappears, which is consequence of an efficient strategy adopted by the government in placing a correct $\pi(S)$ (or the values in $\mathrm{p}_{0}$ and $\mathrm{p}_{1}$ when using the logistic one). The goal is to accomplish convergence, so next lemmas expose a relationship between the degrees of honesty and the booty that might eventually lead to a society free of crime. From now on $l(i, t)=l(i)$ will not depend on t. Let $S^{t}>0$ be the random booty at time $t$.

Lemma 2. Given $\pi: \Re_{\geq 0} \rightarrow[0,1]$ and $t_{0}$, if $\forall t \geq t_{0} H_{i}^{t}>S^{t}$ (in particular, if $H_{i}^{t}>K<W>$ ), then agent $i$ will not commit any crime from instant $t_{0}$.

Lemma 3. If $\exists t_{0} \exists \varepsilon>0 \quad \forall t \geq t_{0} N_{p}{ }^{t}>N_{k}{ }^{t} / c \wedge \forall i \quad\left(\alpha_{i}<1 \Rightarrow \forall t \geq t_{0}\right.$ $H_{i}^{t}-H_{l(i)}{ }^{t}<\delta H_{i}\left(c N_{p}{ }^{t}-N_{k}{ }^{t}\right) /\left(\left(1-\alpha_{i}\right) N_{k}{ }^{t}\right)-\varepsilon$, then crime disappears. 
This offers an interesting view on how to eradicate crime by ensuring the proportion of captures over total crimes, given the leadership and contagion information. We have

Theorem 1. If $\forall i \alpha_{l(i)}=1$ (leaders do not have leaders) $\wedge \exists t_{0} \forall t \geq t_{0} N_{p}{ }^{t}>N_{k}^{t} / c \wedge$ $\forall i\left(\alpha_{i}<1 \Rightarrow \delta H_{i} \leq \delta H_{l(i)} \wedge H_{i}^{t_{0}}-H_{l(i)}^{t_{0}}<\delta H_{i} /\left(\left(1-\alpha_{i}\right) N\right)\right)$, then crime disappears.

That is, for certain conditions regarding differences between initial degrees of honesty and their maximum possible changes, the procedure eventually eradicates crime.

\subsection{Equilibria}

We formulate the problem as a 2-player general sum game [Von Neumann 1947], [Nash 1950] with 1 turn per player as follows: Player 1 is the state, with utility function $u(\pi)$, plays first selecting a probability distribution $\pi$, with the goal of paying as little as possible according to booties as well as the fixed monthly cost of monitoring the agent (and capturing and keeping him in prison if necessary). Player 2 is a fixed agent, who plays second. Given player 1's probability function, he selects any function which, given $S$, decides whether a crime will be committed or not. We may assume or not that $S$ represents money or other good from the state, so there are different possibilities for the damage function. The cost for the state should be monotonically increasing with $\pi$ the apprehension probability, thus it will be reluctant to keep $\pi$ high, ideally optimal when

$$
\pi(S)=\max \left\{\frac{S-H}{2 S+f(S)+\imath \cdot W}, 0\right\}
$$

For simplicity, we omit max leaving only the main term from now on. As soon as $H$ grows, the costs are reduced and conversely. We define the concrete utility for the state, given a distribution $\pi$, in the manner of [Van Eeghen 2016], i.e., previewing a continuum of booties. We have $c(S) \geq 0$ the cost of monitoring a booty $S$ and $d(\pi, S) \geq 0$ a continuous function in $S$ denoting the damage caused by a crime, where $d(\pi, S)=0$ iff there is no crime, otherwise $d(\pi, S)=d(S)$ i.e. $d$ just depends on $S$. Therefore, the state looks forward to maximizing his utility defined as $u(\pi)=-\int(c(s) \pi(s)+d(\pi, s)) d s$ subject to the agent's decision, where the integral goes from $s=0$ to the maximum possible booty (finite or not). The state would support small crimes in order to keep the total cost as low as possible, given the monitoring cost and the damages associated to crimes. A natural assumption is that it is less expensive to monitor an amount of money than to let it be stolen, so this will be a condition in the following

Theorem 2. If $d(\pi, S) \geq c(S)$ for all $S$ such that $d(\pi, S)>0$, then there exists a Nash equilibrium given by $\pi$ as in (4) and the agent not committing crimes for any $S$.

Proof:

$$
\sup _{\pi}-\int(c(s) \pi(s)+d(\pi, s)) d s=-\inf _{\pi}\left(\int c(s) \pi(s) d s+\int d(\pi, s) d s\right)
$$

Since $\int d(\pi, s) d s>0$ when there exists $S$ such as that there is a crime situation, the continuity of $d$ implies that the second term above is optimal when $d(\pi, S)=0$ for all $S$, and of all such $\pi$ the one satisfying equality (4) gives the optimal for the first term. Any deviation will decrease his utility, thus the agent should not commit crimes for any $S$.

Next we search for a single apprehension probability function for the state of the above form, to be applied during instants $0,1, \ldots, T-1$ for one agent whose behavior will depend on the current instant's threshold function (which basically depends on his degree of honesty) and the state's pre-selected $\pi$ function. The best of those functions where the cost for the state is minimized over the whole time interval is, at any instant $t$, 
$\pi_{t}(S)=\frac{S-H^{t}}{2 S+f(S)+1 \cdot W}$. For simplicity, let $\quad H^{t}=H^{0}+\delta H \cdot \frac{t}{T} \quad$ for $t=0,1, \ldots, T-1$

that is, we sort the agent's emerging degrees of honesty and discard repetitions, i.e., fluctuations. Note that $t<t^{\prime}$ implies $\pi_{t}(S) \geq \pi_{t}^{\prime}(S)$ for all $S$.

Theorem 3. To determine the optimal instant $p$ for the threshold function $\pi_{p}$, it is sufficient to find $0 \leq p<T$ which minimizes the function $I: / N \rightarrow / R$ given by

$I(p)=T \int c(s) \pi_{p}(s) d s+p \int d(s) d s$

and set $\pi(s)=\pi_{p}(s)+\varepsilon$ (an infinitesimal is added to ensure the inequality in (4)).

Intuition behind $I(p)$ : the left term decreases with $p$ while the right term increases with $p$. The state's fixed best strategy with the above form is at every point $S$ infinitesimally greater than some $\pi_{p}(S)$, which does not mean an equilibrium for the whole process but a best policy when a single choice of probability distribution is needed. This infimum is algorithmic by the finiteness of $T$ (provided that the above integrals are computable).

Theorem 4. Given two agents with the same $\delta H$ and wage $W$, if $\tau W \geq 1$ then $\forall p$ $\forall H_{1}{ }^{0}, H_{2}{ }^{0}$ respective initial degrees of honesty $\left|I_{1}(p)-I_{2}(p)\right| \leq T \mid H_{1}{ }^{0}-H_{2}{ }^{o} \int c(s) d s$.

\section{Conclusion}

We have extended a crime and punishment model, going from an expected utility function for an optimization process to a Nash equilibrium involving such a utility, where the "moral" equilibrium idea is transferred to a global strategy. Moreover, the last theorems suggest how to learn from the behavior of one agent to handle others. We have studied a (restricted) form of leadership which propagates degrees of honesty. For the future it would be interesting to allow a major interaction between the agents, to study a game with a higher number of players and to find the best estimates for $H$ and $W$ in (4). Another direction is to treat partially unknown degrees of honesty and varying salaries.

\section{References}

Aprea, M., Arbiser, A., Monge, M. (2018). On a model of crime and punishment dynamics. Proc. CACIDI 2018, IEEE. ISBN 978-1-5386-5448-4. https://ieeexplore.ieee.org/document/8584375

Becker, C. (1968). Crime and Punishment: An Economic Approach, Journal of Political Economy, vol. 76, pp. 169-217.

Friedland, N., Maital, S., Rutenberg, A. (1978). A simulation study of income tax evasion. Journal of Public Economics 10, pp. 107-116.

Iglesias, J. R., Semeshenko, V., M. Schneider E. M. and Gordon, M. B. (2012). Crime and punishment: Does it pay to punish? Physica A: Statistical Mechanics and its Applications, vol. 391 issue 15, pp. 3942-3950, August.

Nash, J. F. (1950). Equilibrium points in n-person games, Proc. Nat. Acad. Sci. Wash. 36.

Van Eeghen, W. and Fernández, R. (2016). How to audit income tax - two models combined, Utrecht University, January.

Von Neumann, J. and Morgenstern, O. (1947). Theory of Games and Economic Behavior, Princeton University Press. 